\title{
GAMYB and BPBF Transcriptional Factors in the Control of Gene Expression During Development of Barley Endosperm
}

Isabel Díaz, Jesús Vicente-Carbajosa, Zamira Abraham, Manuel Martínez, Inés Isabel-LaMoneda and Pilar Carbonero

Laboratorio de Bioquímica y Biología Molecular, Departamento de Biotecnología-UPM, ETSI Agrónomos, Ciudad Universitaria s/n, 28040 Madrid, Spain

\section{Introduction}

During cereal seed development, the starchy endosperm cells accumulate proteins, mainly prolamines, as well as several antimicrobial peptides and inhibitors of heterologous hydrolases implicated in plant defence (Shewry et al., 1995; Carbonero et al., 1999). The genes encoding the storage proteins are coordinately expressed in the developing endosperm where they are under spatial and temporal transcriptional control, involving cis-acting motifs in their promoters and trans-acting transcriptional factors (TFs). A number of consensus sequences in gene promoters have been shown to be involved in conferring endosperm specificity in cereals (Albani et al., 1997; Marzabal et al., 1998; Mena et al., 1998; Vicente-Carbajosa et al., 1998; Oñate et al., 1999; Carbonero et al., 2000; Wu et al., 2000; Díaz et al., 2002). A conserved cis-acting motif found in most storage protein gene promoters of seeds is the endosperm-box, a bipartite motif located $\sim 300 \mathrm{bp}$ upstream of the translation initiation codon that contains two distinct binding sites: the GCN4-like motif (GLM) motif (5'-ATGAG/CTCAT-3') that resembles the yeast GCN4 motif and the prolamine box (PB: 5'-TGTAAAG-3'). There is a third motif (5'-AACA/TA-3'), which plays a role in the expression of genes encoding glutelins in rice (Suzuki et al., 1998). These three types of motifs are the target sites of different TFs.

In barley, the GLM is recognized by bZIP proteins of the Opaque 2 family (BLZ1 and BLZ2) that through dimer formation, as either homo- or heterodimers (Vicente-Carbajosa et al., 1998; Oñate et al., 1999), trans-activate the expression of endosperm-specific genes such as the Hor2 and Itr1 genes 
encoding the B-hordeins and a trypsin inhibitor, respectively. The prolamine box is recognized by a TF of the DOF class, DNA binding with $\underline{\text { One Finger }}$ (BPBF), that activates transcription of a native Hor2 promoter in co-bombarded barley endosperms through binding to the prolamine box (PB) (Mena et al., 1998; Carbonero et al., 2000). We describe here how the $5^{\prime}$-AACA/TA-3' motif interacts with a TF of the MYB class, which was previously shown to have a role in the activation of gibberellin (GA)-induced genes upon germination in barley aleurone cells (Gubler et al., 1995, 1999).

\section{Materials and Methods}

\section{Plant material}

Barley (Hordeum vulgare) cv. Bomi was grown in a greenhouse at $18^{\circ} \mathrm{C}$ under 18/6 h day/night photoperiod. Developing endosperms (10-22 days after flowering), mature embryos and 7-day-old leaves and roots were used for RNA extraction. For transient expression assays, developing endosperms collected at 18 days after flowering (DAF) were used immediately.

\section{Northern blot analysis}

RNA was isolated by the Lagrimini et al. (1987) procedure, electrophoresed, blotted to Hybond membranes and hybridized according to standard procedures (Sambrook et al., 1989). Specific labelled probes were used to identify the HvGAMyb, Pbf, Hor2 and Itr1 mRNAs (Díaz et al., 1995; Gubler et al., 1995; Mena et al., 1998).

\section{DNA constructs and particle bombardment in developing endosperms}

The effector constructs contained the cDNAs of GAMYB (Gubler et al., 1995) or $B P B F$ (Mena et al., 1998) driven by the 35S promoter plus the first intron of the alcohol dehydrogenase I gene $(A d h I)$. The reporter constructs included the GUS reporter gene ( $u i d A$ ) fused to the Itr 1 and Hor2 gene promoter fragments (Díaz et al., 1995; Mena et al., 1998), or their mutated versions (see Fig. 9.3a), which corresponded to base changes in the specific MYB and DOF binding motifs.

Particle bombardment was carried out with a biolistic helium gun device (DuPont PDS-000, BioRad, USA). Gold particle coating and bombardment conditions were performed according to Vicente-Carbajosa et al. (1998).

\section{Yeast two-hybrid system assays}

Translational fusions with the GAMYB and BPBF (or derived fragments of it) to the alcohol dehydrogenase I $(A d h I)$ promoter fused to the Gal4 DNAbinding and DNA-activation domains (BD- and $\mathrm{AD}$ fusions, respectively) were done (see Fig. 9.4a). Saccharomyces cerevisiae transformation was per- 


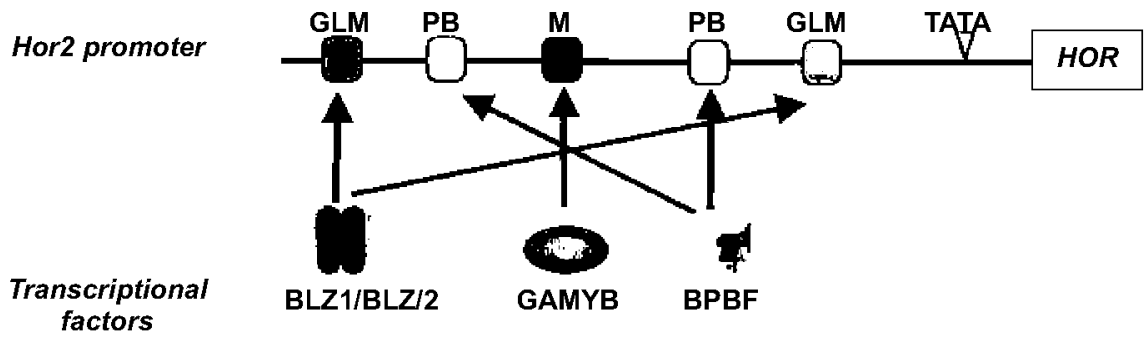

Fig. 9.1. Schematic representation of cis-motifs in the barley Hor2 gene promoter and their putative interaction with transcriptional factors (TF) of the bZIP (BLZ1 and BLZ2), DOF (BPBF) and MYB (GAMYB) classes. GLM: GCN4-like motif; PB: Prolamine Box; M: MYB binding site; TATA: TATA box.

formed by the polyethyleneglycol method and transformants were screened for $\beta$-galactosidase production ( $\mathrm{LaCZ}$ ).

\section{Results and Discussion}

Functional analyses of the promoters of several genes expressed in barley endosperm, such as those encoding B-hordeins (Hor2) and trypsin inhibitor CMe (Itr1), had shown the presence of putative bZIP, MYB and DOF binding motifs in their promoters (Fig. 9.1), which led us to investigate the possible involvement of the HvGAMYB and the BPBF protein factors in their regulation.

The HvGAMYB is a TF of the MYB class that binds specifically to the GA-responsive element (GARE) present in the promoters of $\alpha$-amylases, $\beta$-glucanases and cysteine-proteases of the cathepsin B and cathepsin L types (Cercos et al., 1999; Gubler et al., 1999). Mutations in the GARE abolished both binding and trans-activation. The BPBF is a TF of the DOF class that we had previously shown to be expressed in the developing barley endosperm. The BPBF protein expressed in bacteria was able to bind specifically to the PB motif in a Hor2 gene promoter and through direct interaction with this cis-element did transiently trans-activate transcription from the Hor2 promoter in microprojectile-bombarded developing barley endosperms (Mena et al., 1998; Carbonero et al., 2000).

The expression of the encoding gene GAMYB was analysed by Northern blot and these experiments demonstrated that this gene was already present in developing endosperms at $10 \mathrm{DAF}$ and could be detected throughout all stages analysed, as occurred with the DOF transcription factor BPBF ( $P b f$ gene) (Fig. 9.2). The transcripts of B-hordein (Hor2 gene) and trypsin inhibitor CMe (Itr1 gene) were detectable at $10 \mathrm{DAF}$ and peaked at 22 DAF (Fig. 9.2). The pattern of GAMYB accumulation was consistent with its being a regulator of the Hor 2 and Itr 1 genes, whose temporal mRNA expression overlaps with that of the HvGAMYB. In situ hybridization studies have localized the spatial expression of the $\operatorname{HvGAMYB}$ within the developing 


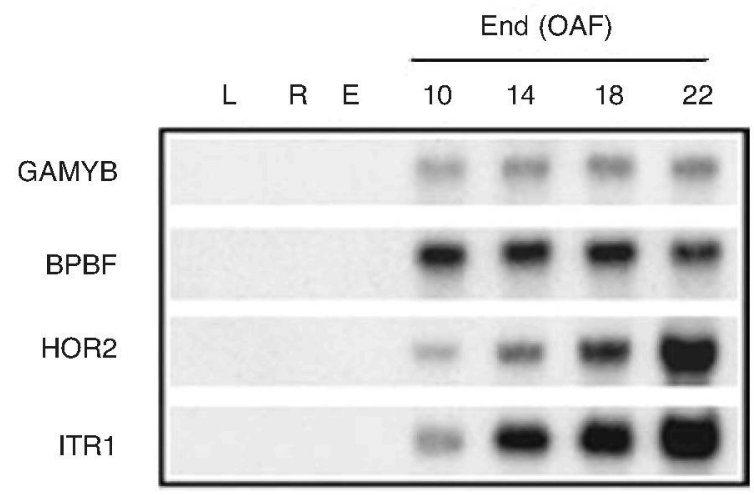

Fig. 9.2. Northern blot analysis of $G A M Y B$ and $B P B F$ mRNA expression in different barley tissues. Total RNA $(8 \mu \mathrm{g})$ from leaves $(\mathrm{L})$, roots $(\mathrm{R})$, mature embryo $(\mathrm{E})$ and developing endosperm (End) at 10-22 DAF were electrophoresed and blotted. The filter was hybridized with specific probes from GAMYB, BPBF, Hor2 and Itr 1 CDNAs.

seed in the endosperms, aleurone layers, vascular tissues and embryo (Díaz et al., 2002).

The capability of in vitro binding of the GAMYB protein to the $5^{\prime}-\mathrm{C} / \mathrm{TAACT} / \mathrm{AACC} / \mathrm{A}-3^{\prime}$ motif in the promoters of Hor 2 and Itr1 genes was shown by electrophoretic mobility shift assays (EMSA), using two oligonucleotide probes whose sequences were deduced from the corresponding regions of the Hor 2 and Itr1 gene promoters and protein extracts derived from Escherichia coli expressing GAMYB as a fusion protein. Retarded bands were produced only when the probes were incubated with the transformed bacterial protein extracts (data not shown). Binding specificity was demonstrated by competition titration up to $100 \times$ concentration with the same unlabelled probe, but not by the mutated probes which differed only in two base changes at the binding MYB motif. The formation of the shifted DNA-protein complexes was not observed with the mutated variants.

The functional relevance of the interaction in vitro was further tested in planta by transient expression assays in co-bombarded developing endosperms (Fig. 9.3). When a reporter gene under the control of the $-343 \mathrm{bp}$ or -560 bp promoter regions from the Hor 2 and Itr1 genes, respectively, containing intact MYB and DOF binding sites (pItr1 and pBhor in Fig. 9.3A), were co-transfected with GAMYB or BPBF as effectors, a threefold to tenfold increase in GUS activity was observed (Fig. 9.3B,C). As expected, mutations in the MYB binding motif (pItr1* and pBhor** in Fig. 9.3A) that prevent in vitro binding by the GAMYB protein in EMSA assays abolished GUS transactivation (Fig. 9.3B,C). In contrast, when the reporter construct mutated in the DOF binding site was used (pBhor* in Fig. 9.3C), GAMYB failed to transactivate GUS expression, although the MYB site was intact, while the BPBF protein, as effector, was still able to increase the GUS activity over the control without effector (Fig. 9.3C), probably because there are two DOF binding sites in the promoter (see Fig. 9.1) participating in the regulation process. 
(A) Effectors

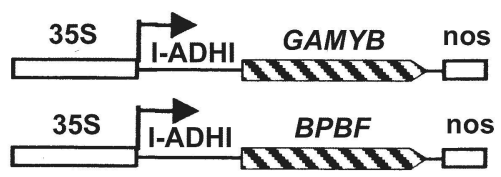

Reporters

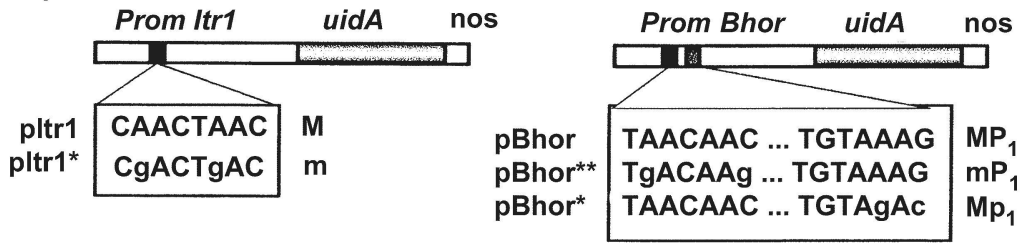

(B)

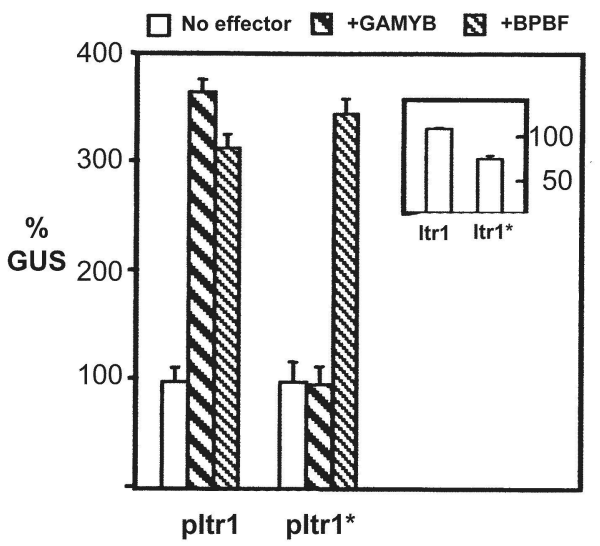

(C)

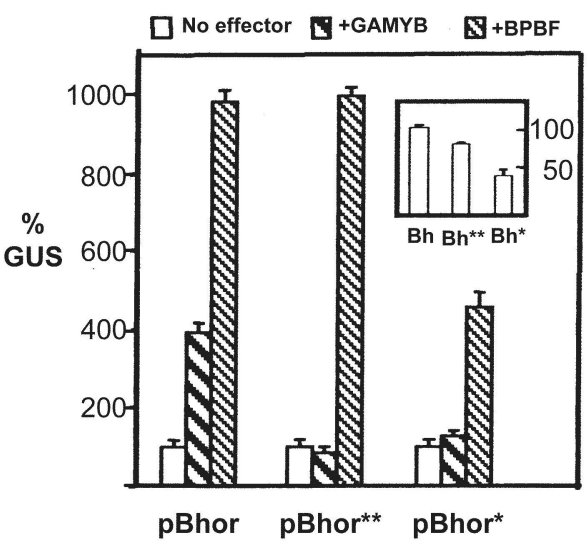

Fig. 9.3. Trans-activaton of the Itr1 and Hor2 promoters by HvGAMYB and BPBF in developing endosperm. (A) Schematic representation of the effector and reporter constructs. The effectors contained the GAMYB or BPBF CDNAs driven by the CaMV 355 promoter plus the first intron of the $A d h /$ gene. The reporter constructs consisted of the GUS gene (uidA) under the control of the Itr1 gene promoter (pltr1), the Hor 2 promoter (pBhor), or under the mutated promoters with the indicated base changes (pltr1*, pBhor* and pBhor**). (B), (C) Transient expression assays by co-bombardment of developing barley endosperms with the indicated combinations of reporter and effector constructs. GUS activity was expressed as percentage relative to the control plasmids without effector. Transient expression driven by the promoter mutants under study relative to their respective wild types are shown in the inserts.

Mutation at the MYB and DOF binding sites in the reporter plasmids resulted in the loss of the basal transcriptional activity of the native pBhor and pItr1 promoter constructs (inserts of Fig. 9.3B,C).

Taken together, all these results indicate that the GAMYB mediates the trans-activation of the Itr 1 and Hor 2 gene promoters in developing barley endosperm through binding to the MYB motif, and that an interaction with the DOF factor BPBF is necessary for the full trans-activation of Hor2. This 
hypothesis was checked, in vivo, by the yeast two-hybrid system, using the effector constructs schematically represented in Fig. 9.4A. As shown in Fig. 9.4B (lane 2), yeast cells co-transformed with the full-length cDNA fragments of both proteins activated the expression of a $L a c$ reporter gene, indicating interaction between these two TFs in this system. This interaction took place between HvGAMYB and the C-terminal part of the BPBF (lane 6 in Fig. 9.4B). In addition, we observed that BPBF is a transcriptional activator in yeast and this protein factor devoid of the N-terminal DOF domain was a stronger activator than the full-length BPBF (lanes 1 and 5 in Fig. 9.4B), an observation previously reported by Kang and Singh (2000) with other DOF transcriptional factors.

These results strongly implicate the GAMYB protein from barley as a TF involved in the combinatorial regulation of genes specifically expressed in the endosperm during development. Recent data from our laboratory have demonstrated that BPBF also has a role in the control of gene expression upon germination (Mena et al., 2002), which suggests that involvement of the same TFs in the processes of seed development and germination may be a more general phenomenon than previously suspected.

\section{Acknowledgements}

Financial support from the Spanish Ministerio de Ciencia y Tecnología (project BMC2000-1483) and from Comunidad Autónoma de Madrid (07G/0015/2000) is gratefully acknowledged.

(A)

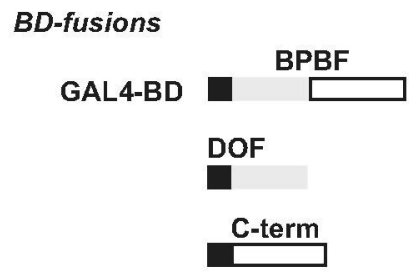

AD-fusions

GAL4-AD
(B)

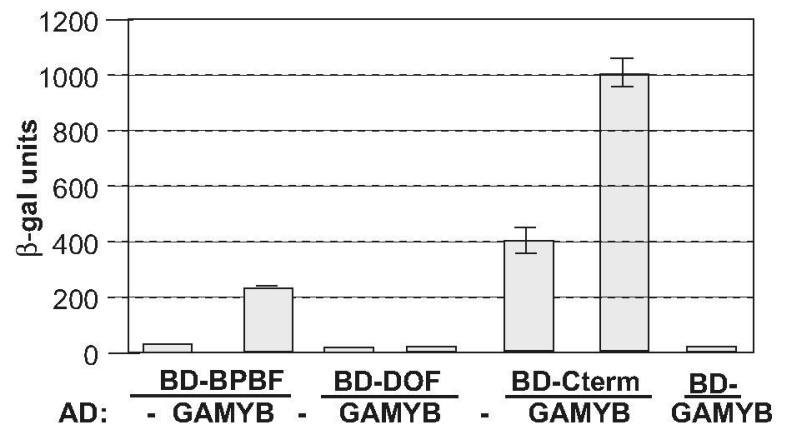

Fig. 9.4. Interaction between HvGAMYB and BPBF in the yeast $S$. cerevisiae two-hybrid system. (A) GAL4-BD and GAL4-AD: GAL4-binding and -activation domains; BPBF: full-length BPBF CDNA (nt 1-999); DOF: BPBF N-terminal region (nt 1-273); C-term: BPBF C-terminal region (nt 274-999); GAMYB: full-length GAMYB CDNA (nt 1-1719). (B) Quantitative evaluation of the $\beta$-galactosidase ( $\operatorname{LaC} Z$ ) reporter activity in liquid medium, expressed as Miller's units. Standard errors of the mean of four isolates were $10 \%$. The reporter $L a c Z$ was driven by GAL4 truncated promoter under the control of Gal4-responsive elements. 


\section{References}

Albani, D., Hammond-Kosack, M.C.U., Smith, C., Conlan, S., Colot, V., Holdsworth, M. and Bevan, M.W. (1997) The wheat transcriptional activator SPA: a seed-specific bZIP protein that recognizes the GCN-4-like motif in the bifactorial endosperm box of prolamin genes. The Plant Cell 9, 171-184.

Carbonero, P., Díaz, I., Vicente-Carbajosa, J., Alfonso-Rubí, J., Gaddour, K. and Lara, P. (1999) Cereal $\alpha$-amylase/trypsin inhibitors and transgenic insect resistance. In: Scarasccia-Mugnozza, G.T., Porceddu, E. and Pagnotta, M.A. (eds) Genetics and Breeding for Crop Quality and Resistance. Kluwer Academic Publishers, Dordrecht, The Netherlands, pp. 147-158.

Carbonero, P., Vicente-Carbajosa, J., Mena, M., Oñate, L., Lara, P. and Diaz, I. (2000) bZIP and DOF transcription factors in the regulation of gene expression in barley endosperm. In: Black, M., Bradford, K.J. and Vazquez-Ramos, J. (eds) Seed Biology. CAB International, Wallingford, UK, pp. 27-41.

Cercos, M., Gomez-Cadenas, A. and Ho, T.H.D. (1999) Hormonal regulation of a cysteine proteinase gene, EPB-1, in barley aleurone layers: cis- and trans-acting elements involved in the co-ordinated gene expression regulated by gibberellins and abscisic acid. The Plant Joumal 19, 107-118.

Diaz, I., Royo, J., O' Connor, A. and Carbonero, P. (1995) The promoter of the Itr1 gene from barley confers different tissue specificity in transgenic tobacco. Molecular General Genetics 248, 592-298.

Diaz, I., Vicente-Carbajosa, J.V., Abraham, Z., Martínez, M., Isabel-LaMoneda, I. and Carbonero, P. (2002) The GAMYB protein from barley interacts with the DOF transcription factor $\mathrm{BPBF}$ and activates endosperm-specific genes during seed development. The Plant Journal 29, 453-464.

Gubler, F., Kalla, R., Roberts, J.K. and Jacobsen, J.V. (1995) Gibberellin-regulated expression of a $m y b$ gene in barley aleurone cells: evidence for Myb transactivation of a High-pI $\alpha$-amylase gene promoter. The Plant Cell 7, 1879-1891.

Gubler, F., Raventos, D., Keys, M., Watts, R., Mundy, J. and Jacobsen, J.V. (1999) Target genes and regulatory domains of the GAMYB transcription activator in barley aleurone. The Plant Journal 17, 1-9.

Kang, H.-G. and Singh, K.B. (2000) Characterization of salicylic acid-responsive Arabidopsis Dof domain proteins: over expression of $O B O 3$ leads to growth defects. The Plant Journal 21, 329-339.

Lagrimini, L.M., Burkhart, W., Moyer, M. and Rosthein, S. (1987) Molecular cloning of complementary DNA encoding the lignin forming peroxidases from tobacco: molecular analysis and tissue-specific expression. Proceedings of the National Academy of Sciences USA 84, 7542-7546.

Marzabal, P., Busk, P.K., Ludevid, M.D. and Torrent, M. (1998) The bifactorial endosperm box of $\gamma$-zein gene: characterization and function of the $\mathrm{Pb} 3$ and GZM cis-acting elements. The Plant Journal 16, 41-52.

Mena, M., Vicente-Carbajosa, J., Schmidt, R.J. and Carbonero, P. (1998) An endosperm-specific DOF protein from barley, highly conserved in wheat, binds to and activates transcription from the prolamin-box of a native B-hordein promoter in barley endosperm. The Plant Journal 16, 53-62.

Mena, M., Cejudo, F.J., Isabel-Lamoneda, I. and Carbonero, P. (2002) A role for the DOF transcription factor BPBF in the regulation of gibberellin-responsive genes in barley aleurone. Plant Physiology 130, 111-119.

Oñate, L., Vicente-Carbajosa, J., Lara, P., Díaz, I. and Carbonero, P. (1999) Barley BLZ2, a seed-specific bZIP protein that interacts with BLZ1 in vivo and activates 
transcription from the GCN4-like motif of B-hordein promoters in barley endosperm. Journal of Biological Chemistry 274, 9175-9182.

Sambrook, J., Fritsch, E.F. and Maniatis, T. (1989) Molecular Cloning: a Laboratory Manual, 2nd edn. Cold Spring Harbor Laboratory Press, Cold Spring Harbor, New York.

Shewry, P.R., Naiser, J.A. and Tatham, A.S. (1995) Seed storage proteins: structure and biosynthesis. The Plant Cell 7, 945-956.

Suzuki, A., Wu, C.Y., Washida, H. and Takaiwa, F. (1998) Rice MYB protein OSMYB5 specifically binds to the AACA motif conserved among promoters of genes for storage protein glutelin. Plant Cell Physiology 39, 555-559.

Vicente-Carbajosa, J., Oñate, L., Lara, P., Díaz, I. and Carbonero, P. (1998) Barley BLZ1: a bZIP transcriptional activator that interacts with endosperm-specific gene promoters. The Plant Journal 13, 629-640.

Wu, C.-Y., Washida, H., Onodera, Y., Harada, K. and Takaiwa, F. (2000) Quantitative nature of the prolamine-box, ACGT and AACA motifs in a rice glutelin gene promoter: minimal cis-element requirements for endosperm-specific gene expression. The Plant Journal 23, 415-421. 